\title{
Real-Time Ultrasonic Tracking of an Intraoperative Needle Tip with Integrated Fibre-Optic Hydrophone
}

\author{
Christian Baker*, Miguel Xochicale*, Francois Joubert*, Fang-yu Lin*, Sunish Mathews ${ }^{\dagger}$, \\ Dzhoshkun Ismail Shakir*, Sebastien Ourselin*, Anna David ${ }^{\dagger}$, Brian Dromey ${ }^{\dagger}$, Adrien Desjardins ${ }^{\dagger}$, \\ Edward Zhang ${ }^{\dagger}$, Paul Beard ${ }^{\dagger}$, Tom Vercauteren*, Wenfeng Xia* \\ ${ }^{*}$ King's College London, London, U.K., ${ }^{\dagger}$ University College London, London, U.K.
}

\begin{abstract}
We have developed a real-time ultrasound needle tracking system based on a fibre-optic hydrophone integrated into an intraoperative needle. The system is intended to track the location of a needle tip during minimally invasive surgical procedures that rely on handheld ultrasound guidance. During these procedures, clear visibility of the needle tip is essential to reach the procedure target and avoid adverse events due to erroneous needle placement. The ultrasound emitted by the handheld probe is detected by the hydrophone and used to draw a cursor marking the position of the needle tip within the $B$ mode ultrasound image. The tracking accuracy was assessed by comparison to manual labelling of the location of the needle tip within the ultrasound images and found to be $\pm 2.2 \mathrm{~mm}$.
\end{abstract}

Index Terms-Needle tracking, real-time, fibre-optic hydrophone, minimally-invasive surgery

\section{INTRODUCTION}

Many minimally invasive surgical procedures rely on ultrasound (US) guidance for accurate insertion of the intraoperative needle, primarily in the contexts of tumour biopsy, regional anaesthesia and fetal medicine. Clear visibility of the needle tip within the US image is essential to reach the procedure target and prevent erroneous needle placement, which can lead to adverse events including nerve damage and pregnancy loss [1]. Needle localisation is often difficult within high-echogenicity tissues, and visibility is dependent on the insertion angle due to specular reflection [2].

Several passive methods have been proposed to improve needle visibility, including those employing image processing algorithms [3], US beam steering [4] and modification of a needle's surface to enhance diffuse scattering [5]. While these methods have been reported to be effective to some extent-and surface modified needles have seen success in the clinic-reliable, clear and accurate localisation of the needle tip still poses a challenge, particularly in high echogenicity tissues [6], at steep insertion angles and in heterogeneous tissues containing structures resembling needle shafts [7].

Active needle tracking methods have also been proposed, typically relying on either an electromagnetic (EM) [8] or US [9]-[12] sensor embedded within the needle tip. An externally generated EM or US field-registered to the US imaging plane-is detected by the sensor, and the acquired signals are used to locate the position of the needle tip within

This work was supported by core and project funding from Wellcome (WT101957; 203148/Z/16/Z; 203145Z/16/Z;), and EPSRC (NS/A000027/1; NS/A000049/1; NS/A000050/1). the field and therefore the US image. EM methods have been shown to be sensitive to environmental field disturbancesfor example from surgical instruments [13] —and provide a positional resolution of $3 \mathrm{~mm}$ or worse [7]. Encouragingly, it has been shown that US tracking has the potential to provide sub-millimetre resolution [12].

We have previously described US tracking methods that require the generation of a separate, external US field [14]; that provide US tracking of the needle tip using only the handheld US field [11], [12]; and that utilise a photoacoustic transmitter at the needle tip [15]. The latter two methods required control of the US pulse sequence, and therefore none were directly compatible with commercial systems.

We have now developed a real-time US needle tracking (UNT) system that can be appended to a clinical US system requiring no external fields, only limited proprietary knowledge of the US transmission sequence and no modifications to the clinical system other than the provision of a line trigger signal. Waveforms received by a fibre-optic hydrophone (FOH) integrated into the needle tip are acquired alongside a feed of the B-mode US video from the clinical system. The location of the needle tip within each frame is determined from the waveforms and marked with a superimposed 'cross hairs' cursor, before the feed is displayed on an external monitor. This paper assesses the accuracy of the system by comparison to manual labelling of the location of the needle tip within the US images.

\section{UNT SYSTEM}

\section{A. Hardware}

The UNT system was developed as an adjunct to a GE (Zift, Austria) Voluson E10 clinical US imaging system with a 'C1-5-D' convex US probe. An FOH is integrated into a 20 gauge, $150 \mathrm{~mm}$ long needle cannula intended for chorionic villus sampling (CVS), detecting the US field emitted by the probe [16]. Signals acquired from the FOH system are used to determine the location of the needle tip within the images generated by the US system.

The clinical US system is configured to operate in B-mode with a beam angle pf $55^{\circ}$ and an imaging depth of $30 \mathrm{~cm}$ as this provides a simple US pulse sequence. The centre frequency of the transmitted acoustic pulses is $2.13 \mathrm{MHz}$ and the US frame rate is $18 \mathrm{~Hz}$. 
The UNT system software runs on a workstation PC sitting alongside the US system. A frame grabber (DVI2PCIe Duo, Epiphan Systems, Ottawa, Canada) acquires the video feed from the US system's HDMI output and transfers it to the UNT software, which superimposes a cursor onto the each US frame at the tracked location of the needle tip and displays the feed on the monitor with a latency of less than $200 \mathrm{~ms}$.

The FOH system was manufactured at University College London and is described in [16]. The FOH was glued into the CVS needle cannula with its sensitive element aligned to the needle tip. A digitiser (M2p.5961-x4, Spectrum Instrumentation $\mathrm{GmbH}$, Großhansdorf, Germany) acquires the voltage waveforms generated by the $\mathrm{FOH}$ console with a sample rate of $10 \mathrm{MHz}$. Acquisitions are triggered by a signal generated by the US system, via a modified transducer cable. During each US frame, a pulse waveform is received from each transducer aperture and stored in a column of a $2 \mathrm{D}$ array in the UNT software. These 2D data structures are referred to as 'FOH frames', where the $n^{\text {th }}$ column in the array contains a waveform representing the acoustic pulse received from the $n^{\text {th }}$ transducer aperture. Each column therefore relates to a scan-line at a single angle $\theta$.

\section{B. Tracking}

Determination of the needle tip position within a US frame takes place in four stages: $\mathrm{FOH}$ waveform preprocessing; $\mathrm{FOH}$ frame gating; determination of the needle tip position within the FOH frame; and conversion of the determined position into coordinates within the acquired US frame.

1) FOH waveform preprocessing: The $\mathrm{FOH}$ waveforms are preprocessed with a matched filter and an envelope detector to improve the performance of the tracking algorithm. The matched filter was designed to maximise the signal-to-noise ratio (SNR), using an approximation of the shape of the received US pulse as a template signal: a Gaussian-windowed sinusoidal pulse at the centre frequency. The length and frequency of the pulse were chosen by examination of the received FOH signals as well as proprietary knowledge provided by GE. A sinusoidal pulse was chosen so that the matched filter would perform equally well across the whole imaging plane, where received pulses have different harmonic content due to non-linear propagation and attenuation. The effect of the matched filter on a representative FOH waveform is shown in Figure 1 The envelope detector was designed to reduce jitter in time-of-flight determination by providing a rectified, singlepeak waveform rather than a pulse containing multiple peaks. The envelope detector consists of a rectifier followed by a 3rd-order Butterworth low-pass filter with a cut-off frequency equal to half of the expected centre frequency of the pulse. The effect of the envelope detector on a representative $\mathrm{FOH}$ waveform is shown in Figure 1 .

2) FOH frame gating: The UNT system was designed to track the needle tip within the US imaging plane, only displaying a cursor if the needle tip was deemed to be located within the imaging plane. The two-dimensional nature of the system and tracking algorithm prevents determination of the
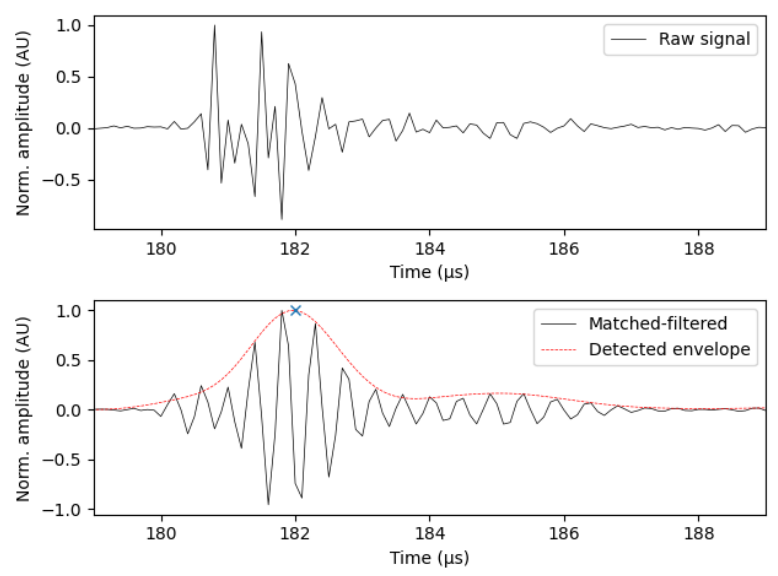

Fig. 1. An example of a low-SNR FOH waveform before preprocessing (top), after processing with the matched filter (bottom, solid black line) and after processing with the envelope detector (bottom, red dotted line). The peak of the envelope, used to measured the time-of-flight of the pulse, is marked with a blue cross.

elevational position of the needle tip; the acoustic pressure and therefore the FOH signal-to-noise ratio (SNR) reduces rapidly as the needle moves away from the imaging plane. The likelihood that the needle tip is in-plane is therefore assessed from the SNR of the preprocessed FOH frames. This requires dividing each frame into 'signal' and 'noise' regions. The 'signal' region is taken to be a rectangle around the peak sample in the frame. The size of the region was chosen based on examination of the maximum US beam width in the imaging plane, and the pulse length. The SNR is approximated by dividing the peak sample value in the frame by the RMS of the values outside of the rectangular region. FOH frames with an SNR below a threshold value, chosen heuristically, are assumed to have been generated with the needle tip outside of the imaging plane and are therefore discarded.

3) Locating the needle-tip within the FOH frame: The location of the needle tip is first determined within the $\mathrm{FOH}$ frame space, in polar coordinates $(r, \theta)$ relative to the centre of curvature of the US probe (see Figure 3). In many cases, this can be estimated simply by taking the $(r, \theta)$ coordinates of the maximum amplitude pixel of the FOH frame. However, in the acoustic near-field this may lead to erroneous localisation due to side-lobes and off-axis maxima. Instead, the angle of the needle tip is found by calculating the energy in each received pulse and then taking the centre-of-mass (CM) of the distribution of energy by angle, as shown in Figure 2. The $\mathrm{CM}$ is found after applying a $-6 \mathrm{~dB}$ threshold to the pulse energy distribution, so that noise does not bias the resulting $\mathrm{CM}$ in either angular direction. If the decibel SNR in the distribution is lower than the negated decibel threshold (i.e. $6 \mathrm{~dB}$ ), then the thresholding fails to prevent $\mathrm{CM}$ bias. The SNR is therefore estimated by dividing the peak energy by the RMS of the energy values outside of a $13^{\circ}$ wide measurement region as shown in Figure 2. The SNR measurement region is centred around the angle with the peak energy, and its width 


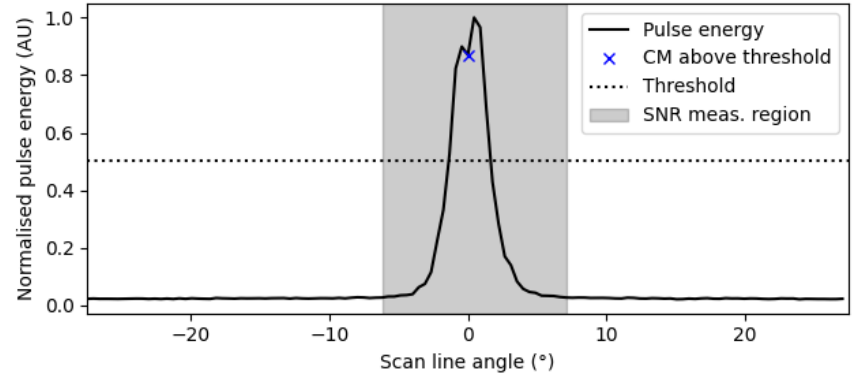

Fig. 2. Plot of the pulse energy received by the hydrophone from each US scan line at a representative near-field location. The CM threshold is plotted as a horizontal dotted line. The resulting $\mathrm{CM}$ is plotted as a cross marker. It can be seen that, in the near field, the CM does not necessarily correspond to the scan line with the maximum energy. The SNR measurement region is shown shaded in grey.

was chosen from observation of $\mathrm{FOH}$ frames received across the US imaging plane. If the estimated SNR is too low, then the $\theta$ coordinate of the max amplitude pixel is used instead. The $r$ coordinate is determined from the time-to-peak $t$ of the waveform in the corresponding column of the $\mathrm{FOH}$ frame: $r=c t+\rho$ where $c$ is the sound speed assumed by the clinical US system and $\rho$ is the radius of curvature of the probe.

4) Coordinate transform: Polar coordinates $(r, \theta)$ are converted to Cartesian coordinates $(x, y)$ relative to the centre of curvature of the probe: $x=r \cos (\theta)$ and $y=r \sin (\theta)$. In order to plot the resulting Cartesian coordinates onto the US frames, it was necessary to determine the size of the pixels of the US frames and the coordinates of the centre of curvature of the probe within the US frames. These parameters were determined automatically through the acquisition and analysis of a single high-gain US frame within which the borders of the fan shaped scan area were visible. The two straight edges of the scan area were parameterised using a straight line Hough transform, and their intersection taken to be the centre of curvature of the probe. The bottom arc of the scan area was parameterised using a circle Hough transform, and the intersections of the resulting arc with the two straight edges were stored. The distances between the centre of curvature and each of the two intersections were assumed to be equal to the US imaging depth selected on the US system, allowing determination of the pixel size.

\section{Software}

The tracking algorithm described in II-B was implemented as part of the UNT system software, written in Python (version 3.8). The UNT software is a real-time desktop application that acquires the US and FOH frames, carries out the tracking, superimposes the needle tip position onto the US frame and displays the modified US frame to the user. The software is capable of real-time tracking at the required frame rate of $18 \mathrm{~Hz}$. The software has the ability to stream raw $\mathrm{FOH}$ and US frames to disk so that they may be used as a data-source at a later date. It also includes a manual labelling feature for

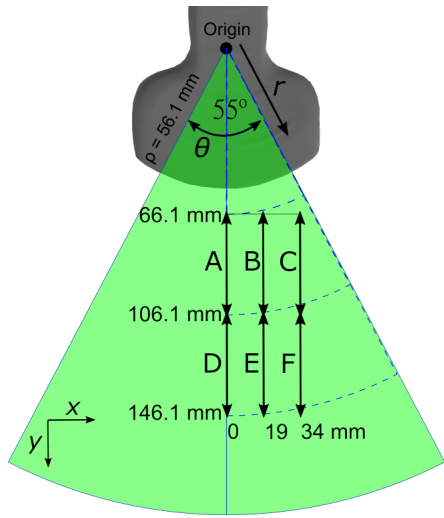

Fig. 3. Diagram showing the regions over which the needle tip was translated for each of the 6 datasets labelled A-F. Coordinates are Cartesian, relative to the centre of curvature of the probe (labelled as 'origin'). For each dataset, the needle was translated from the top of its corresponding arrow on the diagram to the bottom, and then back up to the top.

marking 'true' locations of the needle tips within a previously recorded dataset.

\section{TRACKING ACCURACY EXPERIMENT}

\section{A. Materials}

Tracking accuracy was assessed in a $70 \times 40 \times 40 \mathrm{~cm}$ tank filled with room temperature water. The US probe was mounted pointing vertically downwards with only its faced immersed in the water, to a depth of $10 \mathrm{~mm}$. The needle with integrated FOH was held horizontally beneath the probe, with its long axis parallel to the US imaging plane. The relative orientations of the needle and probe were set by observation of the image of the needle generated by the US system. The needle was mounted on a computer-controlled 3-axis motion control system so that the position of the needle tip could be translated within the imaging plane. The clinical US system was configured as described in II-A with a focal depth of $14 \mathrm{~cm}$ and an acoustic output of $98 \%$.

\section{B. Data collection}

Six datasets were collected with the needle undergoing translation by the motion control system. Each dataset was recorded using the raw data recording feature of the UNT software. During each recording, the needle was translated vertically downwards by $40 \mathrm{~mm}$ and then returned to its original position, reaching a maximum speed of $5 \mathrm{~mm} \mathrm{~s}^{-1}$. The starting positions were set in a grid covering the nearfield of the right hand side of the imaging plane, as shown in Figure 3. Each dataset recording took $27 \mathrm{~s}$, and therefore contained approximately $500 \mathrm{FOH}$ frames and 500 US frames.

\section{Manual labelling}

The 'true' position of the needle tip within the US frames of each of the recorded datasets was manually labelled using the UNT software user interface. The true positions labelled in each datasets were then plotted in order to identify the segments of the recordings during which the needle tip was 


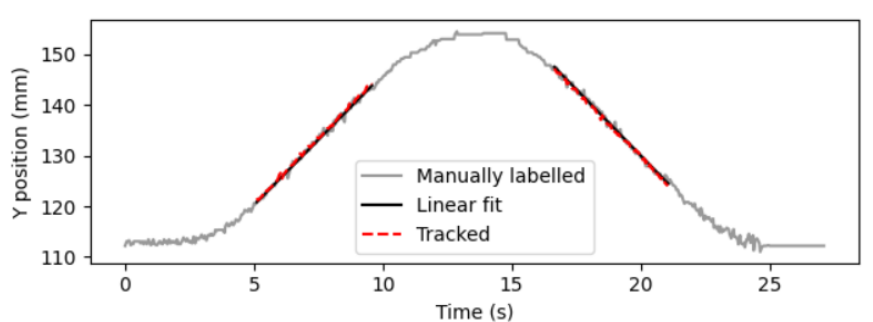

Fig. 4. Plot of the manually labelled and tracked vertical $(y)$ positions from a representative dataset (dataset ' $C$ ' in Figure 3). The linear fits applied to the manually labelled positions in the constant-velocity sections of the dataset are also shown.

moving at a constant velocity. The $x$ and $y$ coordinates of these constant velocity segments were then fitted with straight lines using linear regression, and the accuracy of the manual labelling process was estimated from the mean-square-error (MSE) of the fit. This process is shown in Figure 4

\section{Comparing tracked and labelled positions}

Accuracy was assessed during the constant velocity segments of the recorded datasets: approximately 1000 positions. Vector displacement was calculated between each tracked position and the corresponding true coordinates provided by the linear fits. The mean and standard deviation of displacement in both polar and Cartesian coordinates were then calculated.

\section{REsults}

The MSE of the linear regressions was $0.4 \pm 0.2 \mathrm{~mm}$ in the $x$ direction and $0.3 \pm 0.1 \mathrm{~mm}$ in the $y$ direction. This was used as a proxy for the variance of manual labelling, indicating its accuracy. The mean and standard deviation of the vector displacement between the tracked and manually labelled positions are shown in Table I in both polar and Cartesian coordinates. The mean and standard deviation of the distance between the tracked and labelled positions is also shown.

\section{Discussion AND CONCLUSION}

The estimated variance of the manually labelled data was found to be lower than the variance seen in the tracked positions, indicating that the manually labelled data was accurate enough to be used for comparison. No significant bias was found to be present between the tracked and manually labelled positions. The standard deviation of the tracked lateral $(x)$ coordinates is more than twice that of the tracked depth $(y)$ coordinates. This is reflected in the high standard deviation in the tracked angular $(\theta)$ coordinates, which is equivalent to an uncertainty of approximately \pm 2 scan lines. This suggests that

TABLE I

DISPLACEMENT BETWEEN TRACKED AND LABELLED POSITIONS

\begin{tabular}{|l|l|l|}
\hline Cartesian disp. & Polar disp. & Distance \\
\hline$x=0.1 \pm 2.1 \mathrm{~mm}$ & $\theta=0.0 \pm 0.9^{\circ}$ & $0.2 \pm 2.2 \mathrm{~mm}$ \\
$y=-0.2 \pm 0.8 \mathrm{~mm}$ & $r=-0.3 \pm 0.8 \mathrm{~mm}$ & \\
\hline
\end{tabular}

a major source of angular (and lateral) tracking is quantisation due to scan-line spacing. The standard deviation in the radial direction is approximately equal to the acoustic wavelength, suggesting this as a major source of uncertainty for axial localisation. These results demonstrate that in a homogenous medium in vitro, the UNT system is capable of accurately determining the position of the needle in real-time.

\section{REFERENCES}

[1] K. Agarwal and Z. Alfirevic, "Pregnancy loss after chorionic villus sampling and genetic amniocentesis in twin pregnancies: a systematic review," Ultrasound Obstet Gynecol, vol. 40, no. 2, pp. 128-134, Jul 2012.

[2] J. W. Kim and S. S. Shin, "Ultrasound-guided percutaneous core needle biopsy of abdominal viscera: Tips to ensure safe and effective biopsy," Korean J Radiol, vol. 18, no. 2, p. 309, 2017.

[3] M. I. Daoud, R. N. Rohling, S. E. Salcudean, and P. Abolmaesumi, "Needle detection in curvilinear ultrasound images based on the reflection pattern of circular ultrasound waves," Med Phys, vol. 42, no. 11, pp. 6221-6233, Nov 2015.

[4] S. Cheung and R. Rohling, "Enhancement of needle visibility in ultrasound-guided percutaneous procedures," Ultrasound Med Biol, vol. 30, no. 5, pp. 617-624, May 2004.

[5] P. E. Bigeleisen, A. Hess, R. Zhu, and A. Krediet, "Modeling, production, and testing of an echogenic needle for ultrasound-guided nerve blocks," J Ultrasound Med, vol. 35, no. 6, pp. 1319-1323, Jun 2016.

[6] S. Hebard and G. Hocking, "Echogenic technology can improve needle visibility during ultrasound-guided regional anesthesia," Reg Anesth Pain Med, vol. 36, no. 2, pp. 185-189, Mar 2011.

[7] P. Beigi, S. E. Salcudean, G. C. Ng, and R. Rohling, "Enhancement of needle visualization and localization in ultrasound," Int J Comput Assist Radiol Surg, vol. 16, no. 1, pp. 169-178, Sep 2020.

[8] A. Hakime, F. Deschamps, E. G. M. De Carvalho, A. Barah, A. Auperin, and T. De Baere, "Electromagnetic-tracked biopsy under ultrasound guidance: Preliminary results," Cardiovasc Intervent Radiol, vol. 35, no. 4, pp. 898-905, Aug 2012.

[9] J. J. Langberg, J. O. Franklin, J. S. Landzberg, J. M. Herre, L. Kee, M. C. Chin, S. Bharati, M. Lev, R. B. Himelman, N. B. Schiller, J. C. Griffin, and M. M. Scheinman, "The echo-transponder electrode catheter: A new method for mapping the left ventricle," J Am Coll Cardiol, vol. 12, no. 1, pp. 218-223, Jul 1988.

[10] D. Vilkomerson and D. Lyons, "A system for ultrasonic beacon-guidance of catheters and other minimally-invasive medical devices," IEEE Trans. Ultrason., Ferroelectr., Freq. Control, vol. 44, no. 2, pp. 496-504, Mar 1997.

[11] W. Xia, J. M. Mari, S. J. West, Y. Ginsberg, A. L. David, S. Ourselin, and A. E. Desjardins, "In-plane ultrasonic needle tracking using a fiberoptic hydrophone," Med Phys, vol. 42, no. 10, pp. 5983-5991, 2015.

[12] W. Xia, Y. Ginsberg, S. J. West, D. I. Nikitichev, S. Ourselin, A. L. David, and A. E. Desjardins, "Coded excitation ultrasonic needle tracking: An in vivo study," Medical Physics, vol. 43, no. 7, pp. 4065-4073, Jun 2016.

[13] F. Poulin and L. P. Amiot, "Interference during the use of an electromagnetic tracking system under OR conditions," J Biomech, vol. 35, no. 6, pp. 733-737, 2002.

[14] W. Xia, S. J. West, M. C. Finlay, J.-M. Mari, S. Ourselin, A. L. David, and A. E. Desjardins, "Looking beyond the imaging plane: $3 \mathrm{~d}$ needle tracking with a linear array ultrasound probe," Sci Rep, vol. 7, no. 1, Jun 2017.

[15] W. Xia, S. Noimark, S. Ourselin, S. J. West, M. C. Finlay, A. L. David, and A. E. Desjardins, "Ultrasonic needle tracking with a fibreoptic ultrasound transmitter for guidance of minimally invasive fetal surgery," in Lecture Notes in Computer Science. Springer International Publishing, 2017, pp. 637-645.

[16] S. J. Mathews, S. Mosse, W. Xia, D. I. Shakir, Y. Kunpalin, E. Z. Zhang, P. C. Beard, S. J. West, A. L. David, and A. E. Desjardins, "Ultrasonic needle tracking with a high-finesse fabry-perot fiber optic hydrophone (conference presentation)," in Advanced Biomedical and Clinical Diagnostic and Surgical Guidance Systems XVII, A. Mahadevan-Jansen, Ed. SPIE, Mar 2019. 